\title{
AN IMPULSIVE NONLINEAR SINGULAR VERSION OF THE GRONWALL-BIHARI INEQUALITY
}

NASSER-EDDINE TATAR

Received 11 August 2005; Revised 18 October 2005; Accepted 20 October 2005

We find bounds for a Gronwall-Bihari type inequality for piecewise continuous functions. Unlike works in the prior literature, here we consider inequalities involving singular kernels in addition to functions with delays.

Copyright (c) 2006 Nasser-Eddine Tatar. This is an open access article distributed under the Creative Commons Attribution License, which permits unrestricted use, distribution, and reproduction in any medium, provided the original work is properly cited.

\section{Introduction}

In this paper, we are concerned with the following impulsive integral inequality:

$$
\begin{gathered}
u(t) \leq a(t)+b(t) \int_{0}^{t} k_{1}(t, s) u^{m}(s) d s \\
+c(t) \int_{0}^{t} k_{2}(t, s) u^{n}(s-\tau) d s+d(t) \sum_{0<t_{k}<t} \eta_{k} u\left(t_{k}\right), \quad t \geq 0, \\
u(t) \leq \varphi(t), \quad t \in[-\tau, 0], \tau>0,
\end{gathered}
$$

where $a(t), b(t), c(t)$, and $d(t)$ are nonnegative continuous functions, $m, n>1, \eta_{k} \geq 0$, the points $t_{k}$ (called "instants of impulse effect") are in the increasing order, and $\lim _{k \rightarrow \infty} t_{k}=$ $+\infty$. The kernels $k_{i}(t, s), i=1,2$, are of the form

$$
k_{i}(t, s)=(t-s)^{\beta_{i}-1} s^{\gamma_{i}} F_{i}(s), \quad i=1,2,
$$

where $\beta_{i}>0, \gamma_{i}>-1, F_{i}(t), i=1,2$, and $\varphi(t)$ are nonnegative continuous functions. For this reason, we say that we are in the presence of an impulsive nonlinear singular version of the Gronwall inequality with delay.

We would like to find bounds for solutions to this inequality in the space of piecewise continuous functions $u: X \rightarrow Y\left(X \subset \mathbb{R}, Y \subset \mathbb{R}^{N}\right)$, with points of discontinuity of the first 
kind at the points $t_{k} \in X$. Our functions will also be assumed to be left continuous at the points $t_{k}$. This space will be denoted by $P C(X, Y)$.

Integral inequalities are an important tool to investigate some qualitative and quantitative properties of solutions to differential equations such as existence, uniqueness, boundedness, and stability. Among these integral inequalities, we cite the famous Gronwall inequality and its different generalizations (see $[3,13])$.

Impulsive integral equations, impulsive integro-differential equations, and impulsive differential equations arise naturally in various fields such as population dynamics and optimal control (see the monographs $[2,9,15]$ ). It seems that the first treatment of impulsive systems goes back to the monograph by Krylov and Bogolyubov [8].

The following impulsive integral inequality:

$$
u(t) \leq a+\int_{c}^{t} b(s) u(s) d s+\sum_{c<t_{k}<t} \eta_{k} u\left(t_{k}\right), \quad t \geq 0,
$$

has been first used by Samoilenko and Perestyuk [14] to investigate problems of the form

$$
\begin{array}{cc}
x^{\prime}=f(t, x), & t \neq t_{k}, \\
\Delta x=I_{k}(x), & t=t_{k} .
\end{array}
$$

Then, a similar inequality with constant delay was considered by Bainov and Hristova in [1]. Recently, Hristova in [5] treated a more general inequality with nonlinear functions in $u$. However, in all previous works, the functions (kernels) involved in the integrals are regular, even in the case of integrals of convolution or nonconvolution types (see $[3,13])$.

In this work, we consider the case of singular kernels of the form (1.2). The type of inequalities we are going to discuss arise for instance when we study impulsive evolution problems of the form

$$
\begin{gathered}
\frac{d u}{d t}+A u=f\left(t, u, u_{t}\right), \quad t>0, t \neq t_{k}, \\
u(0)=u_{0} \in X, \\
\Delta u\left(t_{k}\right)=u\left(t_{k}^{+}\right)-u\left(t_{k}^{-}\right), \quad k=1,2, \ldots,
\end{gathered}
$$

where $A$ is a sectorial operator (see, for instance, [17] where the case without delay and with globally Lipschitzian right-hand side is treated).

We point out here that nonlinear singular versions of the Gronwall-Bihari inequality have been already considered by the present author in $[6,7,10,16]$ and Medved in $[11$, 12] to investigate problems of the form (1.5) and perturbed problems of (1.5) but without impulse effects.

The plan of the paper is as follows. In the next section we present some lemmas and notation which will be needed in the proof of our result. Section 3 contains the statement and proof of our theorem. It is ended with some important remarks.

\section{Preliminaries}

In this section, we prepare some lemmas and notation which we will use in the next section. 
LEMma 2.1. For all $\beta>0$ and $\gamma>-1$,

$$
\int_{0}^{t}(t-s)^{\beta-1} s^{\gamma} d s=C t^{\beta+\gamma}, \quad t \geq 0
$$

where $C=C(\beta, \gamma)=\Gamma(\beta) \Gamma(\gamma+1) / \Gamma(\beta+\gamma+1)$.

LEMMA 2.2. If $\beta, \gamma, \delta>0$, then for any $t>0$,

$$
t^{1-\beta} \int_{0}^{t}(t-s)^{\beta-1} s^{\gamma-1} e^{-\delta s} d s \leq C,
$$

where $C=C(\beta, \gamma, \delta)$ is a positive constant independent of $t$. In fact,

$$
C=\max \left\{1,2^{1-\beta}\right\} \Gamma(\gamma)\left(1+\frac{\gamma}{\beta}\right) \delta^{-\gamma}
$$

See [6] for the proof.

LEMMA 2.3. Let $a, b, K, \psi$ be nonnegative continuous functions on the interval $I=(0, T)$ $(0<T \leq \infty)$, let $\omega:(0, \infty) \rightarrow \mathbb{R}$ be a continuous, nonnegative, and nondecreasing function with $\omega(0)=0$ and $\omega(u)>0$ for $u>0$, and let $A(t):=\max _{0 \leq s \leq t} a(s)$ and $B(t):=\max _{0 \leq s \leq t} b(s)$. Assume that

$$
\psi(t) \leq a(t)+b(t) \int_{0}^{t} K(s) \omega(\psi(s)) d s, \quad t \in I .
$$

Then

$$
\psi(t) \leq H^{-1}\left[H(A(t))+B(t) \int_{0}^{t} K(s) d s\right], \quad t \in\left(0, T_{1}\right)
$$

where $H(v):=\int_{v_{0}}^{v} d \tau / \omega(\tau)\left(v \geq v_{0}>0\right), H^{-1}$ is the inverse of $H$, and $T_{1}>0$ is such that $H(A(t))+B(t) \int_{0}^{t} K(s) d s \in D\left(H^{-1}\right)$ for all $t \in\left(0, T_{1}\right)$.

See $[4]$ or $[3,13]$.

In order to lighten the statement of our result, we adopt the following notation. Let $V(\tau):=1+\int_{0}^{\tau} F_{2}^{2}(s) \varphi^{2 n}(s-\tau) d s, r:=\max \{m, n\}>1, t_{0}:=0$.

For $p$ and $q$ such that $1 / p+1 / q=1$, we define

$$
\begin{aligned}
f_{p}(t): & =\sup \left\{a^{q}(t), C^{q / p}\left(p \beta_{1}-p+1, p \gamma_{1}\right) b^{q}(t) t^{q\left(\beta_{1}+\gamma_{1}\right)-1},\right. \\
& \left.C^{q / p}\left(p \beta_{2}-p+1, p \gamma_{2}\right) c^{q}(t) t^{q\left(\beta_{2}+\gamma_{2}\right)-1}, d^{q}(t)\right\}
\end{aligned}
$$

with $C\left(p \beta_{1}-p+1, p \gamma_{1}\right)$ and $C\left(p \beta_{2}-p+1, p \gamma_{2}\right)$ the constants from Lemma 2.1 , and $T_{p}$ 
4 An impulsive Gronwall-Bihari type inequality

to be the sup of all values of $t$ for which

$$
\begin{aligned}
\sum_{i=1}^{k} \int_{t_{i-1}}^{t_{i}}(i+2)^{(q-1) r} \prod_{j=1}^{i-1}\left(1+(j+2)^{q-1} \eta_{j}^{q} f\left(t_{j}\right)\right)^{r} \\
\quad \times\left\{F_{1}^{q}(s) f^{m}(s)+F_{2}^{q}(s) f^{n}(s-\tau)\right\} d s+(k+3)^{(q-1) r} \\
\quad \times \prod_{j=1}^{k}\left(1+(j+2)^{q-1} \eta_{j}^{q} f\left(t_{j}\right)\right)^{r} \int_{t_{k}}^{t}\left\{F_{1}^{q}(s) f^{m}(s)+F_{2}^{q}(s) f^{n}(s-\tau)\right\} d s<\frac{V(\tau)^{1-r}}{(r-1)} .
\end{aligned}
$$

If $p=q=2$, put $f(t):=f_{2}(t)$ and $T:=T_{2}$.

\section{The bounds}

Without loss of generality, we will suppose that the $t_{k}$ are such that $\tau<t_{k+1}-t_{k} \leq 2 \tau$, $k=0,1,2, \ldots$. For the general case, see Remark 3.2 below.

THEOREM 3.1. Let the above assumptions on the different parameters and functions hold. Suppose that $u$ is in $P C([-\tau,+\infty],[0,+\infty])$ and satisfies (1.1), then

(a) if $\beta_{i}>1 / 2$ and $\gamma_{i}>-1 / 2, i=1,2$, it holds that for $t \in\left(t_{k}, t_{+1}\right]$,

$$
\begin{aligned}
u(t) \leq & {\left[(k+3) f(t) \prod_{l=1}^{k}\left(1+(k+2) \eta_{l}^{2} f\left(t_{l}\right)\right)\right]^{1 / q} } \\
\times & {\left[V(\tau)^{1-r}-(r-1) \sum_{i=1}^{k} \int_{t_{i-1}}^{t_{i}}(i+2)^{r} \prod_{j=1}^{i-1}\left(1+(j+2) \eta_{j}^{2} f\left(t_{j}\right)\right)^{r}\right.} \\
& \times\left\{F_{1}^{2}(s) f^{m}(s)+F_{2}^{2}(s) f^{n}(s-\tau)\right\} d s-(r-1)(k+3)^{r} \\
& \left.\times \prod_{j=1}^{k}\left(1+(j+2) \eta_{j}^{2} f\left(t_{j}\right)\right)^{r} \int_{t_{k}}^{t}\left\{F_{1}^{2}(s) f^{m}(s)+F_{2}^{2}(s) f^{n}(s-\tau)\right\} d s\right]^{1 / 2(1-r)}
\end{aligned}
$$

as long as the expression between the second brackets is positive, that is, on $(0, T)$;

(b) if $0<\beta_{i} \leq 1 / 2$ and $-1<\gamma_{i} \leq-1 / 2$, then it holds that for $t \in\left(t_{k}, t_{+1}\right]$,

$$
\begin{aligned}
u(t) \leq\left[(k+3)^{q-1} f_{p}(t) \prod_{l=1}^{k}\left(1+(k+2)^{q-1} \eta_{l}^{q} f\left(t_{l}\right)\right)\right]^{1 / q} \\
\times\left[V(\tau)^{1-r}-(r-1) \sum_{i=1}^{k} \int_{t_{i-1}}^{t_{i}}(i+2)^{(q-1) r} \prod_{j=1}^{i-1}\left(1+(j+2)^{q-1} \eta_{j}^{q} f\left(t_{j}\right)\right)^{r}\right. \\
\quad \times\left\{F_{1}^{q}(s) f_{p}^{m}(s)+F_{2}^{q}(s) f_{p}^{n}(s-\tau)\right\} d s-(r-1)(k+3)^{(q-1) r} \\
\left.\quad \times \prod_{j=1}^{k}\left(1+(j+2)^{q-1} \eta_{j}^{q} f\left(t_{j}\right)\right)^{r} \int_{t_{k}}^{t}\left\{F_{1}^{q}(s) f_{p}^{m}(s)+F_{2}^{2}(s) f_{p}^{n}(s-\tau)\right\} d s\right]^{1 / q(1-r)}
\end{aligned}
$$

as long as the expression between the second brackets is positive, that is, on $\left(0, T_{p}\right)$. 
Proof. We will use a mathematical induction.

(a) Step 1. We start by proving the validity of (3.1) in the interval $\left[0, t_{1}\right]$ (in fact, the argument we present is valid within the interval $(0, T)$, this fact will be mentioned in every occasion by indicating the right interval over which the estimate is valid). For $t \in$ $[0, \tau] \subset\left[0, t_{1}\right]$ (see assumptions on $t_{k}$ ), we have

$$
\begin{aligned}
u(t) \leq & a(t)+b(t) \int_{0}^{t}(t-s)^{\beta_{1}-1} s^{\gamma_{1}} F_{1}(s) u^{m}(s) d s \\
& +c(t) \int_{0}^{t}(t-s)^{\beta_{2}-1} s^{\gamma_{2}} F_{2}(s) u^{n}(s-\tau) d s
\end{aligned}
$$

If $\beta_{i}>1 / 2$ and $\gamma_{i}>-1 / 2, i=1,2$, then by the Cauchy-Schwarz inequality and Lemma 2.1, we obtain

$$
\begin{aligned}
u(t) \leq & a(t)+C^{1 / 2}\left(2 \beta_{1}-1,2 \gamma_{1}\right) b(t) t^{\beta_{1}+\gamma_{1}-1 / 2}\left(\int_{0}^{t} F_{1}^{2}(s) u^{2 m}(s) d s\right)^{1 / 2} \\
& +C^{1 / 2}\left(2 \beta_{2}-1,2 \gamma_{2}\right) c(t) t^{\beta_{2}+\gamma_{2}-1 / 2}\left(\int_{0}^{t} F_{2}^{2}(s) u^{2 n}(s-\tau) d s\right)^{1 / 2}
\end{aligned}
$$

where $C\left(2 \beta_{1}-1,2 \gamma_{1}\right)$ and $C\left(2 \beta_{2}-1,2 \gamma_{2}\right)$ are the constants from Lemma 2.1. Squaring both sides of (3.4), we find

$$
\begin{aligned}
u^{2}(t) \leq & 3 a^{2}(t)+3 C\left(2 \beta_{1}-1,2 \gamma_{1}\right) b^{2}(t) t^{2\left(\beta_{1}+\gamma_{1}\right)-1} \int_{0}^{t} F_{1}^{2}(s) u^{2 m}(s) d s \\
& +3 C\left(2 \beta_{2}-1,2 \gamma_{2}\right) c^{2}(t) t^{2\left(\beta_{2}+\gamma_{2}\right)-1} \int_{0}^{t} F_{2}^{2}(s) u^{2 n}(s-\tau) d s .
\end{aligned}
$$

Therefore

$$
\begin{aligned}
u^{2}(t) & \leq 3 f(t)\left(1+\int_{0}^{t} F_{1}^{2}(s) u^{2 m}(s) d s+\int_{0}^{t} F_{2}^{2}(s) u^{2 n}(s-\tau) d s\right) \\
& \leq 3 f(t)\left(1+\int_{0}^{t} F_{1}^{2}(s) u^{2 m}(s) d s+\int_{0}^{\tau} F_{2}^{2}(s) \varphi^{2 n}(s-\tau) d s\right)
\end{aligned}
$$

Putting

$$
v_{1}(t):=1+\int_{0}^{\tau} F_{2}^{2}(s) \varphi^{2 n}(s-\tau) d s+\int_{0}^{t} F_{1}^{2}(s) u^{2 m}(s) d s
$$

we see that $v_{1}(t)$ is a nondecreasing positive differentiable function on $[0, \tau], v_{1}(0)=$ $1+\int_{0}^{\tau} F_{2}^{2}(s) \varphi^{2 n}(s-\tau) d s=: V(\tau)$,

$$
\begin{gathered}
u^{2}(t) \leq 3 f(t) v_{1}(t), \\
v_{1}^{\prime}(t)=F_{1}^{2}(t) u^{2 m}(t) \leq 3^{m} F_{1}^{2}(t) f^{m}(t) v_{1}^{m}(t) \leq 3^{r} F_{1}^{2}(t) f^{m}(t) v_{1}^{r}(t) .
\end{gathered}
$$

An integration of (3.9) (or using Lemma 2.3 directly) leads to

$$
v_{1}(t) \leq\left[V(\tau)^{1-r}-3^{r}(r-1) \int_{0}^{t} F_{1}^{2}(s) f^{m}(s) d s\right]^{1 /(1-r)}
$$


6 An impulsive Gronwall-Bihari type inequality

as long as $\int_{0}^{t} F_{1}^{2}(s) f^{m}(s) d s<V(\tau)^{1-r} / 3^{r}(r-1)$. Therefore, for $t \in[0, \tau]$,

$$
u(t) \leq \sqrt{3 f(t)}\left[V(\tau)^{1-r}-3^{r}(r-1) \int_{0}^{t} F_{1}^{2}(s) f^{m}(s) d s\right]^{1 / 2(1-r)}
$$

as long as $\int_{0}^{t} F_{1}^{2}(s) f^{m}(s) d s<V(\tau)^{1-r} / 3^{r}(r-1)$.

Let $t \in\left(\tau, t_{1}\right]$. Then, from (3.6) and (3.7), we have

$$
u^{2}(t) \leq 3 f(t)\left(v_{1}(\tau)+\int_{\tau}^{t} F_{1}^{2}(s) u^{2 m}(s) d s+\int_{\tau}^{t} F_{2}^{2}(s) u^{2 n}(s-\tau) d s\right) .
$$

Let us designate

$$
w_{1}(t):=v_{1}(\tau)+\int_{\tau}^{t} F_{1}^{2}(s) u^{2 m}(s) d s+\int_{\tau}^{t} F_{2}^{2}(s) u^{2 n}(s-\tau) d s
$$

Then $w_{1}(t)$ is a nondecreasing positive differentiable function on $\left(\tau, t_{1}\right]$,

$$
\begin{gathered}
w_{1}(\tau)=v_{1}(\tau) \leq w_{1}(t), \quad u^{2}(t) \leq 3 f(t) w_{1}(t), \\
w_{1}^{\prime}(t)=F_{1}^{2}(t) u^{2 m}(t)+F_{2}^{2}(t) u^{2 n}(t-\tau) .
\end{gathered}
$$

Since $0<t-\tau \leq \tau$ (see Remark 3.2) and from (3.7), (3.8), (3.14), and (3.15),

$$
u^{2}(t-\tau) \leq 3 f(t-\tau) v_{1}(t-\tau) \leq 3 f(t-\tau) v_{1}(\tau) \leq 3 f(t-\tau) w_{1}(t)
$$

and we can write that

$$
\begin{aligned}
w_{1}^{\prime}(t) & \leq F_{1}^{2}(t)\left(3 f(t) w_{1}(t)\right)^{m}+F_{2}^{2}(t)\left(3 f(t-\tau) w_{1}(t)\right)^{n} \\
& \leq 3^{r}\left[F_{1}^{2}(t) f^{m}(t)+F_{2}^{2}(t) f^{n}(t-\tau)\right] w_{1}^{r}(t) .
\end{aligned}
$$

Integrating (3.17) from $\tau$ to $t$ and using (3.10), we obtain

$$
\begin{aligned}
w_{1}(t) \leq & {\left[w_{1}(\tau)^{1-r}-3^{r}(r-1) \int_{\tau}^{t}\left[F_{1}^{2}(s) f^{m}(s)+F_{2}^{2}(s) f^{n}(s-\tau)\right] d s\right]^{1 /(1-r)} } \\
\leq & {\left[V(\tau)^{1-r}-3^{r}(r-1) \int_{0}^{\tau} F_{1}^{2}(s) f^{m}(s) d s\right.} \\
& \left.\quad-3^{r}(r-1) \int_{\tau}^{t}\left[F_{1}^{2}(s) f^{m}(s)+F_{2}^{2}(s) f^{n}(s-\tau)\right] d s\right]^{1 /(1-r)} \\
\leq & {\left[V(\tau)^{1-r}-3^{r}(r-1) \int_{0}^{t}\left[F_{1}^{2}(s) f^{m}(s)+F_{2}^{2}(s) f^{n}(s-\tau)\right] d s\right]^{1 /(1-r)} }
\end{aligned}
$$

and hence, for $t \in\left(\tau, t_{1}\right]$,

$$
u(t) \leq \sqrt{3 f(t)}\left[V^{1-r}-3^{r}(r-1) \int_{0}^{t}\left[F_{1}^{2}(s) f^{m}(s)+F_{2}^{2}(s) f^{n}(s-\tau)\right] d s\right]^{1 / 2(1-r)}
$$


as long as

$$
\int_{0}^{t}\left[F_{1}^{2}(s) f^{m}(s)+F_{2}^{2}(s) f^{n}(s-\tau)\right] d s<\frac{V^{1-r}}{3^{r}(r-1)} .
$$

We define the function $\psi_{1}:\left[0, t_{1}\right] \rightarrow \mathbb{R}$ by

$$
\psi_{1}(t):= \begin{cases}v_{1}(t), & t \in[0, \tau] \\ w_{1}(t), & t \in\left(\tau, t_{1}\right]\end{cases}
$$

It can be easily seen that $(3.1)$ in the statement of the theorem is satisfied over $\left[0, t_{1}\right]$ (recall that $\left.t_{0}:=0\right)$.

Step 2. Let $t \in\left(t_{1}, t_{2}\right]$. If $t \in\left(t_{1}, t_{1}+\tau\right]$, then

$$
\begin{aligned}
u(t) \leq & a(t)+b(t) \int_{0}^{t}(t-s)^{\beta_{1}-1} s^{\gamma_{1}} F_{1}(s) u^{m}(s) d s \\
& +c(t) \int_{0}^{t}(t-s)^{\beta_{2}-1} s^{\gamma_{2}} F_{2}(s) u^{n}(s-\tau) d s+\eta_{1} d(t) u\left(t_{1}\right)
\end{aligned}
$$

Squaring both sides of (3.22) after applying the Cauchy-Schwarz inequality and Lemma 2.1, as in the previous steps from (3.4) to (3.6), we find

$$
\begin{aligned}
u^{2}(t) \leq 4 f(t)(1 & \left.+\int_{0}^{t} F_{1}^{2}(s) u^{2 m}(s) d s+\int_{0}^{t} F_{2}^{2}(s) u^{2 n}(s-\tau) d s+\eta_{1}^{2} u^{2}\left(t_{1}\right)\right) \\
\leq 4 f(t)( & v_{1}(\tau)+\int_{\tau}^{t_{1}} F_{1}^{2}(s) u^{2 m}(s) d s+\int_{\tau}^{t_{1}} F_{2}^{2}(s) u^{2 n}(s-\tau) d s \\
& \left.+\int_{t_{1}}^{t} F_{1}^{2}(s) u^{2 m}(s) d s+\int_{t_{1}}^{t} F_{2}^{2}(s) u^{2 n}(s-\tau) d s+\eta_{1}^{2} u^{2}\left(t_{1}\right)\right) .
\end{aligned}
$$

Note here that we have used definition (3.7) of $v_{1}(t)$. Thanks to (3.13) and (3.14), we entail that

$$
\begin{aligned}
u^{2}(t) & \leq 4 f(t)\left(w_{1}\left(t_{1}\right)+\int_{t_{1}}^{t} F_{1}^{2}(s) u^{2 m}(s) d s+\int_{t_{1}}^{t} F_{2}^{2}(s) u^{2 n}(s-\tau) d s+3 \eta_{1}^{2} f\left(t_{1}\right) w_{1}\left(t_{1}\right)\right) \\
& \leq 4 f(t)\left[1+3 \eta_{1}^{2} f\left(t_{1}\right)\right]\left(w_{1}\left(t_{1}\right)+\int_{t_{1}}^{t} F_{1}^{2}(s) u^{2 m}(s) d s+\int_{t_{1}}^{t} F_{2}^{2}(s) u^{2 n}(s-\tau) d s\right) .
\end{aligned}
$$

We define

$$
v_{2}(t):=w_{1}\left(t_{1}\right)+\int_{t_{1}}^{t} F_{1}^{2}(s) u^{2 m}(s) d s+\int_{t_{1}}^{t} F_{2}^{2}(s) u^{2 n}(s-\tau) d s
$$


8 An impulsive Gronwall-Bihari type inequality

It is clear that $v_{2}(t)$ is a nondecreasing positive differentiable function on $\left(t_{1}, t_{1}+\tau\right]$,

$$
v_{2}\left(t_{1}\right)=w_{1}\left(t_{1}\right) \leq v_{2}(t), \quad u^{2}(t) \leq 4 f(t)\left[1+3 \eta_{1}^{2} f\left(t_{1}\right)\right] v_{2}(t) .
$$

Since $t-\tau \leq t_{1}$, by (3.6), (3.12), (3.13), and (3.25), we see that

$$
u^{2}(t-\tau) \leq 3 f(t-\tau) \psi_{1}(t-\tau) \leq 3 f(t-\tau) w_{1}\left(t_{1}\right) \leq 3 f(t-\tau) v_{2}(t)
$$

and thus from this estimation, (3.25) and (3.26), we get

$$
\begin{aligned}
v_{2}^{\prime}(t) & =F_{1}^{2}(t) u^{2 m}(t)+F_{2}^{2}(t) u^{2 n}(t-\tau) \\
& \leq\left\{4^{m}\left[1+3 \eta_{1}^{2} f\left(t_{1}\right)\right]^{m} f^{m}(t) F_{1}^{2}(t)+3^{n} F_{2}^{2}(t) f^{n}(t-\tau)\right\} v_{2}^{r}(t) .
\end{aligned}
$$

An integration of (3.28) from $t_{1}$ to $t$ together with (3.18) leads to

$$
\begin{aligned}
v_{2}(t) \leq & {\left[v_{2}\left(t_{1}\right)^{1-r}-(r-1)\right.} \\
& \left.\times \int_{t_{1}}^{t}\left\{4^{m}\left[1+3 \eta_{1}^{2} f\left(t_{1}\right)\right]^{m} f^{m}(s) F_{1}^{2}(s)+3^{n} F_{2}^{2}(s) f^{n}(s-\tau)\right\} d s\right]^{1 /(1-r)} \\
\leq & {\left[V(\tau)^{1-r}-3^{r}(r-1) \int_{0}^{t_{1}}\left[F_{1}^{2}(s) f^{m}(s)+F_{2}^{2}(s) f^{n}(s-\tau)\right] d s-(r-1)\right.} \\
& \left.\times \int_{t_{1}}^{t}\left\{4^{m}\left[1+3 \eta_{1}^{2} f\left(t_{1}\right)\right]^{m} f^{m}(s) F_{1}^{2}(s)+3^{n} F_{2}^{2}(s) f^{n}(s-\tau)\right\} d s\right]^{1 /(1-r)}
\end{aligned}
$$

and hence, for $t \in\left(t_{1}, t_{1}+\tau\right]$, we have

$$
\begin{aligned}
u(t) \leq & 2 \sqrt{\left[1+3 \eta_{1}^{2} f\left(t_{1}\right)\right] f(t)} \\
\times & {\left[V(\tau)^{1-r}-3^{r}(r-1) \int_{0}^{t_{1}}\left[F_{1}^{2}(s) f^{m}(s)+F_{2}^{2}(s) f^{n}(s-\tau)\right] d s\right.} \\
& \left.\quad-(r-1) \int_{t_{1}}^{t}\left\{4^{m}\left[1+3 \eta_{1}^{2} f\left(t_{1}\right)\right]^{m} f^{m}(s) F_{1}^{2}(s)+3^{n} F_{2}^{2}(s) f^{n}(s-\tau)\right\} d s\right]^{1 / 2(1-r)}
\end{aligned}
$$

as long as

$$
\begin{aligned}
3^{r} \int_{0}^{t_{1}} & {\left[F_{1}^{2}(s) f^{m}(s)+F_{2}^{2}(s) f^{n}(s-\tau)\right] d s } \\
& +\int_{t_{1}}^{t}\left\{4^{m}\left[1+3 \eta_{1}^{2} f\left(t_{1}\right)\right]^{m} f^{m}(s) F_{1}^{2}(s)+3^{n} F_{2}^{2}(s) f^{n}(s-\tau)\right\} d s \leq \frac{V^{1-r}}{r-1} .
\end{aligned}
$$

Now let $t \in\left(t_{1}+\tau, t_{2}\right]$, then from (3.7), (3.13), (3.14), (3.25), and

$$
u^{2}(t) \leq 4 f(t)\left(1+\int_{0}^{t} F_{1}^{2}(s) u^{2 m}(s) d s+\int_{0}^{t} F_{2}^{2}(s) u^{2 n}(s-\tau) d s+\eta_{1}^{2} u^{2}\left(t_{1}\right)\right),
$$


we deduce that

$$
\begin{aligned}
u^{2}(t) & \leq 4 f(t)\left(v_{2}\left(t_{1}+\tau\right)+\int_{t_{1}+\tau}^{t} F_{1}^{2}(s) u^{2 m}(s) d s+\int_{t_{1}+\tau}^{t} F_{2}^{2}(s) u^{2 n}(s-\tau) d s+3 \eta_{1}^{2} f\left(t_{1}\right) v_{2}\left(t_{1}+\tau\right)\right) \\
& \leq 4 f(t)\left[1+3 \eta_{1}^{2} f\left(t_{1}\right)\right]\left(v_{2}\left(t_{1}+\tau\right)+\int_{t_{1}+\tau}^{t} F_{1}^{2}(s) u^{2 m}(s) d s+\int_{t_{1}+\tau}^{t} F_{2}^{2}(s) u^{2 n}(s-\tau) d s\right)
\end{aligned}
$$

because $w_{1}\left(t_{1}\right) \leq v_{2}\left(t_{1}\right) \leq v_{2}\left(t_{1}+\tau\right)$. At this stage, we denote

$$
w_{2}(t):=v_{2}\left(t_{1}+\tau\right)+\int_{t_{1}+\tau}^{t} F_{1}^{2}(s) u^{2 m}(s) d s+\int_{t_{1}+\tau}^{t} F_{2}^{2}(s) u^{2 n}(s-\tau) d s
$$

Then, clearly $w_{2}(t)$ is a nondecreasing positive differentiable function on $\left(t_{1}+\tau, t_{2}\right]$, $w_{2}\left(t_{1}+\tau\right)=v_{2}\left(t_{1}+\tau\right) \leq w_{2}(t)$, and

$$
w_{2}^{\prime}(t)=F_{1}^{2}(t) u^{2 m}(t)+F_{2}^{2}(t) u^{2 n}(t-\tau)
$$

Observe that by (3.33) and (3.34), we have the estimates

$$
u^{2}(t) \leq 4 f(t)\left[1+3 \eta_{1}^{2} f\left(t_{1}\right)\right] w_{2}(t)
$$

and since $t_{1}<t-\tau<t_{1}+\tau$, it follows from (3.24) that

$$
\begin{aligned}
u^{2}(t-\tau) & \leq 4 f(t-\tau)\left[1+3 \eta_{1}^{2} f\left(t_{1}\right)\right] v_{2}(t-\tau) \\
& \leq 4 f(t-\tau)\left[1+3 \eta_{1}^{2} f\left(t_{1}\right)\right] v_{2}\left(t_{1}+\tau\right) \\
& \leq 4 f(t-\tau)\left[1+3 \eta_{1}^{2} f\left(t_{1}\right)\right] w_{2}(t) .
\end{aligned}
$$

Consequently,

$$
w_{2}^{\prime}(t) \leq 4^{r}\left[1+3 \eta_{1}^{2} f\left(t_{1}\right)\right]^{r}\left\{f^{m}(t) F_{1}^{2}(t)+f^{n}(t-\tau) F_{2}^{2}(t)\right\} w_{2}^{r}(t) .
$$

Again by an integration of (3.38), we end up with

$$
\begin{aligned}
w_{2}(t) \leq & {\left[w_{2}^{1-r}\left(t_{1}+\tau\right)-4^{r}(r-1)\left[1+3 \eta_{1}^{2} f\left(t_{1}\right)\right]^{r} \int_{t_{1}+\tau}^{t}\left\{f^{m}(s) F_{1}^{2}(s)+f^{n}(s-\tau) F_{2}^{2}(s)\right\} d s\right]^{1 /(1-r)} } \\
\leq & {\left[V(\tau)^{1-r}-3^{r}(r-1) \int_{0}^{t_{1}}\left[F_{1}^{2}(s) f^{m}(s)+F_{2}^{2}(s) f^{n}(s-\tau)\right] d s\right.} \\
& \quad-(r-1) \int_{t_{1}}^{t_{1}+\tau}\left\{4^{m}\left[1+3 \eta_{1}^{2} f\left(t_{1}\right)\right]^{m} f^{m}(s) F_{1}^{2}(s)+3^{n} F_{2}^{2}(s) f^{n}(s-\tau)\right\} d s \\
& \left.\quad-4^{r}(r-1)\left[1+3 \eta_{1}^{2} f\left(t_{1}\right)\right]^{r} \int_{t_{1}+\tau}^{t}\left\{f^{m}(s) F_{1}^{2}(s)+f^{n}(s-\tau) F_{2}^{2}(s)\right\} d s\right]^{1 /(1-r)}
\end{aligned}
$$


or simply

$$
\begin{aligned}
w_{2}(t) \leq[ & {\left[(\tau)^{1-r}-3^{r}(r-1) \int_{0}^{t_{1}}\left[F_{1}^{2}(s) f^{m}(s)+F_{2}^{2}(s) f^{n}(s-\tau)\right] d s\right.} \\
& \left.\quad-4^{r}(r-1)\left[1+3 \eta_{1}^{2} f\left(t_{1}\right)\right]^{r} \int_{t_{1}}^{t}\left\{f^{m}(s) F_{1}^{2}(s)+f^{n}(s-\tau) F_{2}^{2}(s)\right\} d s\right]^{1 /(1-r)} .
\end{aligned}
$$

Hence,

$$
\begin{aligned}
u(t) \leq & 2 \sqrt{f(t)\left[1+3 \eta_{1}^{2} f\left(t_{1}\right)\right]} \\
\times & {\left[V(\tau)^{1-r}-3^{r}(r-1) \int_{0}^{t_{1}}\left[F_{1}^{2}(s) f^{m}(s)+F_{2}^{2}(s) f^{n}(s-\tau)\right] d s\right.} \\
& \left.\quad-4^{r}(r-1)\left[1+3 \eta_{1}^{2} f\left(t_{1}\right)\right]^{r} \int_{t_{1}}^{t}\left\{f^{m}(s) F_{1}^{2}(s)+f^{n}(s-\tau) F_{2}^{2}(s)\right\} d s\right]^{1 / 2(1-r)}
\end{aligned}
$$

provided that the expression between brackets is positive. We define $\psi_{2}:\left(t_{1}, t_{2}\right] \rightarrow \mathbb{R}$ by

$$
\psi_{2}(t):= \begin{cases}v_{2}(t), & t \in\left(t_{1}, t_{1}+\tau\right] \\ w_{2}(t), & t \in\left(t_{1}+\tau, t_{2}\right] .\end{cases}
$$

It is clear that $(3.1)$ holds on $\left(t_{1}, t_{2}\right]$.

Step 3. Finally, suppose that (3.1) is valid over $\left(t_{k}, t_{k+1}\right]$, then if $t \in\left(t_{k+1}, t_{k+2}\right]$, we define

$$
\psi_{k+2}(t):= \begin{cases}v_{k+2}(t), & t \in\left[t_{k+1}, t_{k+1}+\tau\right], \\ w_{k+2}(t), & t \in\left(t_{k+1}+\tau, t_{k+2}\right],\end{cases}
$$

with

$$
\begin{gathered}
v_{k+2}(t):=w_{k+1}\left(t_{k+1}\right)+\int_{t_{k+1}}^{t} F_{1}^{2}(s) u^{2 m}(s) d s+\int_{t_{k+1}}^{t} F_{2}^{2}(s) u^{2 n}(s-\tau) d s \\
w_{k+2}(t):=v_{k+2}\left(t_{k+1}+\tau\right)+\int_{t_{k+1}+\tau}^{t} F_{1}^{2}(s) u^{2 m}(s) d s+\int_{t_{k+1}+\tau}^{t} F_{2}^{2}(s) u^{2 n}(s-\tau) d s .
\end{gathered}
$$

In a similar manner as in Steps 1 and 2, we can see that (3.1) is valid over $\left(t_{k+1}, t_{k+2}\right]$.

(b) If $0<\beta_{i} \leq 1 / 2$ and $-1<\gamma_{i} \leq-1 / 2$, then instead of the Cauchy-Schwarz inequality we use the Hölder inequality with

$$
1<p<\min \left\{\frac{1}{1-\beta_{i}},-\frac{1}{\gamma_{i}}, i=1,2\right\},
$$


and $q>1$ such that $1 / p+1 / q=1$. We find

$$
\begin{aligned}
u(t) \leq & a(t)+C^{1 / p}\left(p \beta_{1}-p+1, p \gamma_{1}\right) b(t) t^{\beta_{1}+\gamma_{1}-1 / q}\left(\int_{0}^{t} F_{1}^{q}(s) u^{q m}(s) d s\right)^{1 / q} \\
& +C^{1 / p}\left(p \beta_{2}-p+1, p \gamma_{2}\right) c(t) t^{\beta_{2}+\gamma_{2}-1 / q}\left(\int_{0}^{t} F_{2}^{q}(s) u^{q n}(s-\tau) d s\right)^{1 / q}
\end{aligned}
$$

Then, we raise both sides to the power $q$ and we use the inequality

$$
\left(\sum_{i=1}^{n} a_{i}\right)^{r} \leq n^{r-1}\left(\sum_{i=1}^{n} a_{i}^{r}\right), \quad n \in \mathbb{N}^{*}, r, a_{i} \in \mathbb{R}^{+}, i=1, \ldots, n
$$

The rest of the proof remains the same. The proof is now complete.

Remark 3.2. Apart from the case treated in the proof, that is, when $\tau<t_{k+1}-t_{k} \leq 2 \tau$, $k=0,1,2, \ldots$, there are several other cases, but each and every one of them can fit in the one considered above or one of the following cases.

Case 1. There exists an $n_{k}>1$ such that $t_{k+1}-t_{k} \geq n_{k} \tau$, that is, $t_{k}<t_{k}+n_{k} \tau \leq t_{k+1}$. In this case, we argue in a similar fashion over $\left(t_{k}, t_{k}+\tau\right],\left(t_{k}+\tau, t_{k}+2 \tau\right], \ldots,\left(t_{k}+\left(n_{k}-1\right) \tau, t_{k}+\right.$ $\left.n_{k} \tau\right]$ and then over $\left(t_{k}+n_{k} \tau, t\right]$ with $t>t_{k}+n_{k} \tau$. Therefore the function $\psi_{k+1}(t)$ will have $n_{k}+1$ components.

Case 2. There exists $k_{0} \geq 1$ such that $\left(t_{k_{0}}, t_{k_{0}+1}\right]$ does not contain any $t_{k_{0}}+n \tau, n=1,2, \ldots$, that is, $t_{k_{0}+1}-t_{k_{0}}<\tau$. Here we deal with this interval in a single step using only a function of the form $v_{k_{0}+1}(t)$, that is, $\psi_{k_{0}+1}(t):=v_{k_{0}+1}(t)$.

Case 3. $\tau \in\left(t_{k_{1}}, t_{k_{1}+1}\right]$ with $k_{1}>0$, that is, $\tau \notin\left(0, t_{1}\right]$ as in Case 1 . Again, in this situation, we consider only functions of the form $v_{k}(t)$ until we reach the interval $\left(t_{k_{1}}, t_{k_{1}+1}\right]$ where we consider both $v_{k_{1}+1}(t)$ and $w_{k_{1}+1}(t)$.

Remark 3.3. Obviously, if $k_{i}(t, s)=(t-s)^{\beta_{i}-1} s^{\gamma_{i}} e^{-\delta_{i} s} F_{i}(s), \delta_{i}>0, i=1,2 \ldots$, the proof still works. However, using Lemma 2.2 instead of Lemma 2.1 throughout the proof, we can have much larger intervals over which the estimations are valid.

Remark 3.4. It is clear that our result can be easily extended to other nonlinearities than the polynomial ones, iterated integrals and the case of several variables. One may use the Gronwall-Bihari lemma (Lemma 2.3) in case of a nondecreasing nonlinearity. See also $[3,13]$ for other classes of nonlinearities.

\section{Acknowledgments}

The author is very grateful for the financial support and the facilities provided by King Fahd University of Petroleum and Minerals. Thanks are due also to the anonymous referees. 


\section{References}

[1] D. D. Bainov and S. G. Hristova, Impulsive integral inequalities with a deviation of the argument, Mathematische Nachrichten 171 (1995), 19-27.

[2] D. D. Bainov and P. S. Simeonov, Systems with Impulse Effect: Theory and Applications, Ellis Horwood, Chichister, 1989.

[3] _ Integral Inequalities and Applications, Mathematics and Its Applications, vol. 57, Kluwer Academic, Dordrecht, 1992.

[4] G. Butler and T. Rogers, A generalization of a lemma of Bihari and applications to pointwise estimates for integral equations, Journal of Mathematical Analysis and Applications 33 (1971), no. 1, $77-81$.

[5] S. G. Hristova, Nonlinear delay integral inequalities for piecewise continuous functions and applications, Journal of Inequalities in Pure and Applied Mathematics 5 (2004), no. 4, 1-14, article 88.

[6] M. Kirane and N.-E. Tatar, Global existence and stability of some semilinear problems, Archivum Mathematicum (Brno) 36 (2000), no. 1, 33-44.

[7] _ Convergence rates for a reaction-diffusion system, Zeitschrift für Analysis und ihre Anwendungen. Journal for Analysis and Its Applications 20 (2001), no. 2, 347-357.

[8] N. N. Krylov and N. N. Bogolyubov, Introduction to Nonlinear Mechanics, Izd. Acad. Sci. Ukr. SSR, Kiev, 1937.

[9] V. Lakshmikantham, D. D. Bainov, and P. S. Simeonov, Theory of Impulsive Differential Equations, Series in Modern Applied Mathematics, vol. 6, World Scientific, New Jersey, 1989.

[10] S. Mazouzi and N.-E. Tatar, Global existence for some integro-differential equations with delay subject to non-local conditions, Zeitschrift für Analysis und ihre Anwendungen. Journal for Analysis and Its Applications 21 (2002), no. 1, 249-256.

[11] M. Medved, A new approach to an analysis of Henry type integral inequalities and their Bihari type versions, Journal of Mathematical Analysis and Applications 214 (1997), no. 2, 349-366.

[12] Singular integral inequalities and stability of semilinear parabolic equations, Archivum Mathematicum (Brno) 34 (1998), no. 1, 183-190.

[13] B. G. Pachpatte, Inequalities for Differential and Integral Equations, Mathematics in Science and Engineering, vol. 197, Academic Press, California, 1998.

[14] A. M. Samoilenko and N. A. Perestyuk, Stability of solutions of differential equations with impulse effect, Differential Equations 13 (1977), no. 11, 1981-1992 (Russian).

[15] _ Impulsive Differential Equations, World Scientific Series on Nonlinear Science. Series A: Monographs and Treatises, vol. 14, World Scientific, New Jersey, 1995.

[16] N.-E. Tatar, Exponential decay for a semilinear problem with memory, Arab Journal of Mathematical Sciences 7 (2001), no. 1, 29-45.

[17] W. Zhang, R. P. Agarwal, and E. Akin-Bohner, On well-posedness of impulsive problems for nonlinear parabolic equations, Nonlinear Studies 9 (2002), no. 2, 145-153.

Nasser-Eddine Tatar: Department of Mathematical Sciences, King Fahd University of Petroleum and Minerals, Dhahran 31261, Saudi Arabia

E-mail address: tatarn@kfupm.edu.sa 